\title{
Erratum to: Homogeneous pancreatic cancer spheroids mimic growth pattern of circulating tumor cell clusters and macrometastases: displaying heterogeneity and crater-like structure on inner layer
}

Hao Feng ${ }^{1,2}$ (1) $\cdot$ Bao-chi Ou ${ }^{1} \cdot$ Jing-kun Zhao ${ }^{1} \cdot$ Shuai Yin $^{2} \cdot$ Eva Oechsle $^{2,3}$.

Ai-guo Lu ${ }^{1}$

Published online: 16 June 2017

(c) Springer-Verlag GmbH Germany 2017

\section{Erratum to: J Cancer Res Clin Oncol}

DOI 10.1007/s00432-017-2434-2

In the original publication of the article, the list of coauthors has been incorrectly published. It has been changed by the corresponding authors with approval of all coauthors. The correct author group should be:

Hao Feng, Bao-chi Ou, Jing-kun Zhao, Shuai Yin, Eva Oechsle, Ai-guo Lu.

The online version of the original article can be found under doi:10.1007/s00432-017-2434-2.

Hao Feng

hao.feng@med.uni-muenchen.de

1 Department of Digestive Surgery, Ruijin Hospital, Shanghai Jiao Tong University School of Medicine, 200025 Shanghai, China

2 Medical Faculty, University Hospital of LMU Munich, 81377 Munich, Germany

3 Eurofins BioPharma Product Testing Germany, 82152 Munich, Germany 